\title{
Review on population-based metaheuristic search techniques for optimal power flow
}

\author{
Muhammad Affiq Abd Rahman, Bazilah Ismail, Kanendra Naidu, Mohd Khairil Rahmat \\ Electrical Engineering Section, Universiti Kuala Lumpur British Malaysian Institute, Malaysia
}

\begin{tabular}{|c|c|}
\hline Article Info & ABSTRACT \\
\hline $\begin{array}{l}\text { Keywords: } \\
\text { Heuristic search } \\
\text { Optimal power flow } \\
\text { Optimization } \\
\text { Power system } \\
\text { Swarm intelligence }\end{array}$ & $\begin{array}{l}\text { Optimal power flow (OPF) is a non-linear solution which is significantly } \\
\text { important in order to analyze the power system operation. The use of } \\
\text { optimization algorithm is essential in order to solve OPF problems. } \\
\text { The emergence of machine learning presents further techniques which } \\
\text { capable to solve the non-linear problem. The performance and the key } \\
\text { aspects which enhances the effectiveness of these optimization techniques } \\
\text { are compared within several metaheuristic search techniques. This includes } \\
\text { the operation of particle swarm optimization (PSO) algorithm, firefly } \\
\text { algorithm (FA), artificial bee colony (ABC) algorithm, ant colony } \\
\text { optimization (ACO) algorithm and differential evolution (DE) algorithm. } \\
\text { This paper reviews on the key elements that need to be considered when } \\
\text { selecting metaheuristic techniques to solve OPF problem in power } \\
\text { system operation. } \\
\text { Copyright } \odot 2019 \text { Institute of Advanced Engineering and Science. } \\
\text { All rights reserved. }\end{array}$ \\
\hline \multicolumn{2}{|l|}{ Corresponding Author: } \\
\hline $\begin{array}{l}\text { Bazilah Ismail, } \\
\text { Electrical Engineering Section, } \\
\text { Universiti Kuala Lumpur British Mala } \\
53100 \text { Gombak, Selangor, Malaysia. } \\
\text { Email: bazilahismail@unikl.edu.my }\end{array}$ & ysian Institute, \\
\hline
\end{tabular}

\section{INTRODUCTION}

In recent decades, concentrated research effort were employed to solve complex optimization problems. Several methods are integrated to ensure the techniques able to operate in optimal rate. One of it is optimal power flow (OPF) problem in power system operation. The major concern is to reduce total operation cost while satisfying various constraints in the system. Computational intelligence assists to understand the problems with various perspective by introducing several metaheuristic techniques which are inspired by biological systems [1].

Evolutionary computation is used to find optimal solutions. This includes genetic algorithm (GA) which is used for search and optimization purposes; evolutionary algorithm (EA) which is excellent on decision making; and multi-agent based algorithm which works best on sorting and usually used for data mining. Traditionally, Newton-Raphson method that is used to identify the optimal values which is started by making an initial guess. This method requires large number of iterations to achieve optimal solution for the problems. Modern metaheuristic algorithm improves the process by using randomization to improve the convergence rate. Convergence of an algorithm is crucial to achieve optimal results which might take long time to converge. While reducing the time will lead to premature converge which means the solution provided does not guarantee global optima for the problem that being considered [2].

Several population-based heuristic search techniques are used to provide high quality optimal solution in acceptable timescale. Several changes in parameter setting are made to suit the nature of the optimization technique such as particle swarm optimization (PSO) algorithm, ant colony optimization (ACO) algorithm, artificial bee colony (ABC) algorithm, firefly algorithm (FA) and differential evolution (DE) algorithm. These algorithms used the iterative process and self-organize system to search the space of 
solution using evolutionary operation. Since it is impossible to create a universal algorithm which able to solve variety of optimization problems, researchers have come out with several metaheuristics with similar framework that contains several changes in the parameter. Although it is good to have variety of nature inspired algorithms such as glowworm swarm optimization (GSO) algorithm, monkey algorithm (MA), lion optimization algorithm (LOA), grey wolf optimization (GWO) algorithm and etc, the main focus is the needed to achieve a balanced exploration and exploitation process to achieve complete convergence with reasonable time based on the complexity of the problems. This paper reviews on the key elements that need to be considered to solve OPF problem in power system operation based on five population-based metaheuristic search techniques which are PSO, FA, ABC, ACO and DE.

\section{OPTIMAL POWER FLOW PROBLEM FORMULATION}

The combination of power flow equation and economic dispatch equation can be simplified into non-linear function. Standard OPF problem can be formulated to minimize the objective in the system and satisfy system equality and inequality constrains as:

Minimize $\quad \mathrm{f}(\mathrm{x}, \mathrm{u})$

Subject to $\quad \mathrm{g}(\mathrm{x}, \mathrm{u}) \leq 0$

$$
\mathrm{h}(\mathrm{x}, \mathrm{u})=0
$$

where :

$$
\begin{array}{ll}
\mathrm{x} & : \text { The dependent variables } \\
\mathrm{u} & : \text { The independent variables } \\
\mathrm{f}(\mathrm{x}, \mathrm{u}) & : \text { Objective functions } \\
\mathrm{g}(\mathrm{x}, \mathrm{u}) & : \text { Set of inequality constraints } \\
\mathrm{h}(\mathrm{x}, \mathrm{u}) & \text { : Set of equality constraints }
\end{array}
$$

\subsection{The Dependent Variable (State Vector)}

$$
\mathrm{x}^{\mathrm{T}}=\left[\mathrm{P}_{\mathrm{G} 1}, \mathrm{~V}_{\mathrm{L} 1} \ldots \mathrm{V}_{\mathrm{LNL}}, \mathrm{Q}_{\mathrm{G} 1} \ldots \mathrm{Q}_{\mathrm{GNG}}, \mathrm{S}_{\mathrm{l} 1} \ldots \mathrm{S}_{\mathrm{In} 1}\right]
$$

where : PG1 : Generator active power at slack bus

VL : Load-bus voltage

QG : Generator reactive power

Sl : Transmission line flow

NL : Number of load buses

NG : Number of generators

$\mathrm{nl}$ : Number of transmission lines

$\mathrm{T}$ : Transformer tap setting

\subsection{The Indipendent Variable (Control Vector)}

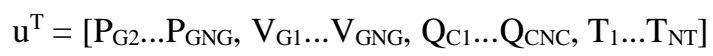

where : PG : Generator active power at PV buses (except at the slack bus PG1)

VG : Generator voltage at PV buses

QC : Shunt VAR compensation

NT : Number of regulating transformers

NC : Number of VAR compensators

\subsection{The Equality Constrains (Typical Load Flow Equation)}

$$
\begin{aligned}
P_{G i}-P_{D i}-V_{i} \sum_{j=1}^{\mathrm{NB}} & V_{j}\left[\mathrm{G}_{\mathrm{ij}} \cos \left(\delta_{\mathrm{i}}-\delta_{\mathrm{j}}\right)\right. \\
& \left.+B_{i j} \sin \left(\delta_{\mathrm{i}}-\delta_{\mathrm{j}}\right)\right]=0
\end{aligned}
$$




$$
\begin{aligned}
Q_{G i}-Q_{D i}-V_{i} \sum_{j=1}^{\mathrm{NB}} V_{j}\left[\mathrm{G}_{\mathrm{ij}} \cos \left(\delta_{\mathrm{i}}-\delta_{\mathrm{j}}\right)\right. & \\
& \left.+B_{i j} \sin \left(\delta_{\mathrm{i}}-\delta_{\mathrm{j}}\right)\right]=0
\end{aligned}
$$

where: NB : Number of buses

PD : Active load demands

QD : Reactive load demands

Gij : Transfer conductance

Bij : Susceptance

\subsection{The Inequality Constrains (Operation Limits of Equipment) Generator constraints:}

$$
\begin{aligned}
& V_{G i}^{\min } \leq V_{G i} \leq V_{G i}^{\max }, \mathrm{i}=1, \ldots, \mathrm{NG} \\
& P_{G i}^{\min } \leq P_{G i} \leq P_{G i}^{\max }, \mathrm{i}=1, \ldots, \mathrm{NG} \\
& Q_{G i}^{\min } \leq Q_{G i} \leq Q_{G i}^{\max }, \mathrm{i}=1, \ldots, \mathrm{NG}
\end{aligned}
$$

Transformer constraints:

$$
T_{i}^{\min } \leq T_{i} \leq T_{i}^{\max }, \mathrm{i}=1, \ldots, \mathrm{NT}
$$

Shunt VAR constraints:

$$
Q_{C i}^{\min } \leq Q_{C i} \leq Q_{C i}^{\max }, \mathrm{i}=1, \ldots, \mathrm{NC}
$$

Security constraints:

$$
\begin{aligned}
& V_{L i}^{\min } \leq V_{L i} \leq V_{L i}^{\max }, \mathrm{i}=1, \ldots, \mathrm{NL} \\
& S_{l i} \leq S_{l i}^{\max }, \mathrm{i}=1, \ldots, \mathrm{nl}
\end{aligned}
$$

The OPF problems solution are designed by considering both normal and contingency operation conditions. This is to ensure that contingencies factor will not cause any violations of limits. Since the inequality constrains are self-limiting therefore, the optimization techniques using evolutionary computation techniques can be performed to overcome the problem.

\section{PERSPECTIVE OF OPTIMIZATION ALGORITHM}

\subsection{Exploitation (Intensification) and Exploration (Diversification)}

Exploitation or intensification in an algorithm is important to generate new solutions which better than existing solutions referring to the problem of interest. This allow high convergence rates but usually trapped in local search. However, the local search is limiting the end solution since it did not compare the information globally and it depends on the starting location. Exploration or diversification on the other hand, search for information globally and able to generate the solution with enough diversity. Since it needs to search in large space, this will slow down the convergence rate [1].

The balance between exploitation and exploration will be able to converge the solution faster and the potential to achieve global optima is high. The current algorithms use different parameter setting which cause certain algorithm to achieve fast convergence rate or able to achieve global optima. Randomization also been used by certain algorithms to make sure the convergence rate is fast. Since there are so many algorithms that perform very well in very specific problem, No Free Lunch (NFL) theorems is introduced. NFL states there is no universal algorithm that efficient enough for every problem. This is because the main purpose of OPF is to obtain high quality solution within acceptable timescale. So there is no reason to obtain average solution for all problems that being considered. However, some researchers suggest that NFL did exist on some types of problems such as coevolutionary approaches [3]. 


\subsection{Evolutionary Operators}

GA and EA uses genetic operators such as recombination (crossover), mutation and selection (survival of fitness) [4]. This allows diversity from mutation and crossover process; and also from the selection and survival of fitness high quality information can be obtained. This is important to solve complex and discontinuous problems. The rate of crossover and mutation will influence the global search rate (exploration) while the selection increase the exploitation action. In general, crossover is used to mix within a subspace and allowing to reach convergence of system. Mutation is a randomization technique which makes the iterative process for local search faster [2]. The selection is used to allow the system to evolve towards desired states. This happen by allowing the current solution compared with previous best solution, if the current solution generate better solution, the previous solution will be discarded. This repetitive process is carried out until the final best solution is achieved. Taxonomy of Evolutionary Computation (Optimization Algorithm) is shown in Figure 1.

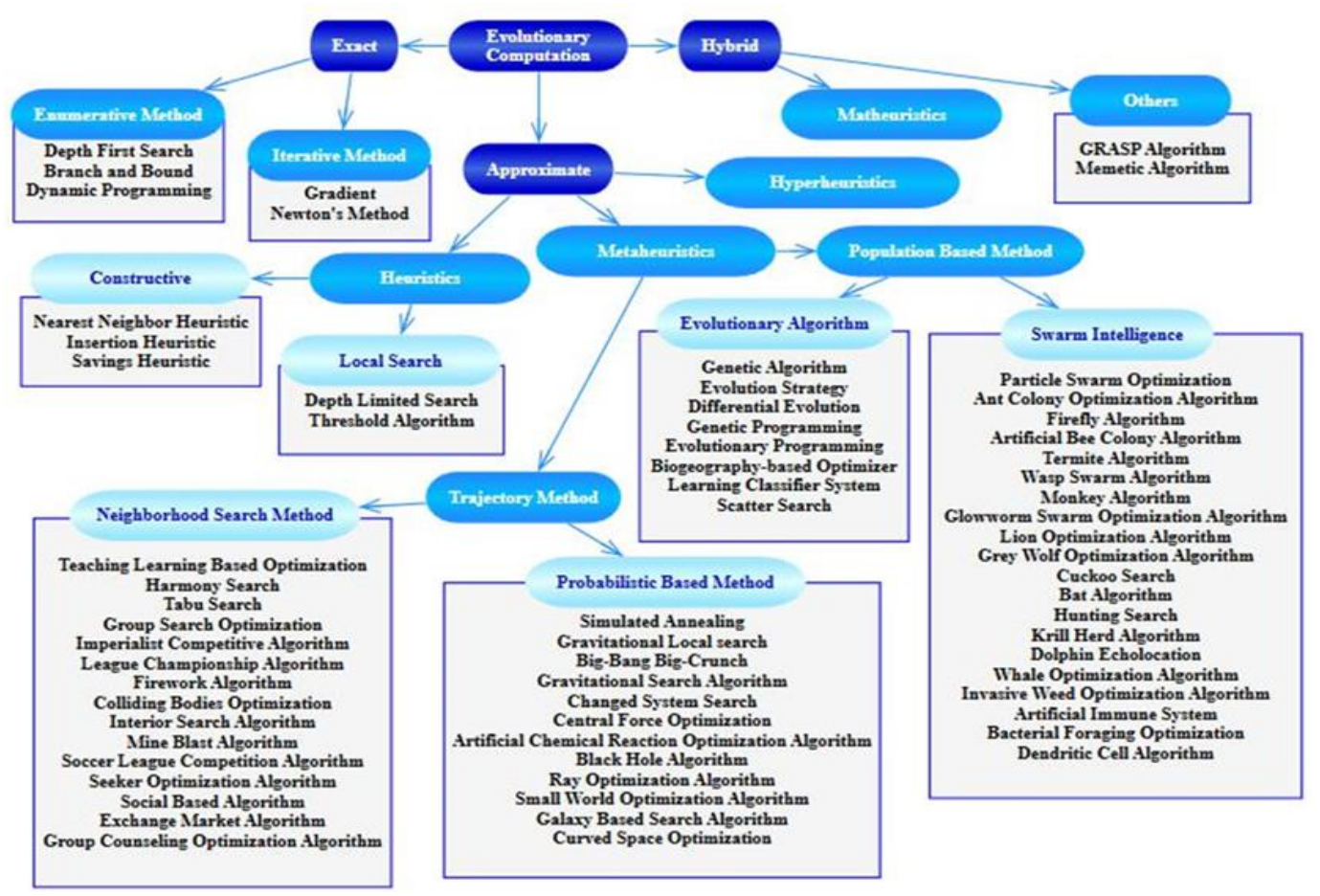

Figure 1. Taxonomy of Evolutionary Computation (Optimization Algorithm)

\subsection{Heuristic Techniques}

Heuristic technique is an approach to solve problem or learning using practical method to obtain satisfactory solution within reasonable time. This occurs by using similar rules from data set which has been trained to solve similar problems. Based on Figure 1, heuristic techniques can be classified into 4 categories which are heuristics, metaheuristics, matheuristics and hyperheuristics.

Matheuristics is a hybrid techniques that combines exact and approximate method to solve mathematical problems of interest using their exploitation features. These techniques uses improved mathematic programming techniques based on metaheuristics and vice versa [5]. This technique also shows significant improvement in local searching and local branching during neighborhood exploration [6], [7].

The approximate techniques which involves heuristics, metaheuristics and hyperheuristics applies exploitation and exploration. However, for heuristics method, it focus more on the exploitation which allows the problem able to obtain good solution in reasonable time. But they are usually trapped in local optima due to minimal exploration.

Metaheuristics techniques are suitable to optimize large and complex data. This is possible due to the behavior which accepts a temporary deterioration of the solution. Active exploration and exploitation allows it to randomly explore to obtain global optimum solution. However, the true balance of exploration and exploitation in any algorithms or problems still does not have a clear line and is supported with NFL theorem. 
Hyperheuristics focus more on exploration compared to exploitation. The set space also not as usual space set of solution. This is because hyperheuristics is used as heuristics to search for optimal heuristics to be used. It is used to handle several classes of problem rather than solving just one problem [8]. The idea is to compensate the strength and weakness of known heuristics to select suitable method to be used for the assigned problems.

\subsection{Classification of Metaheuristics}

There are four main criteria that are used to classify various technique of metaheuristics. Firstly, the method used for every iteration is classified between trajectory and discontinuous methods. Usually trajectory methods is used for single point search in which the process just closed walk on the neighborhood graph. Discontinuous methods commonly used by population based techniques which allows it to make large jumps in the neighborhood graph for high exploration in the search space.

Second criteria is the involvement of memory in the algorithm. Since most of the techniques which is based upon either short term memory or long term memory, hence there is no clear group for this criteria. The purpose of having short term memory is to avoid cycling in similar solution by forbid the revisiting to the recent solution. Long term memory on the other hands, allows good diversification and intensification.

Third criteria is the way the algorithm interact with neighborhood structures. It is either kick-moves operation which interact with single neighbor or using operation that able to interact with various neighborhood such as mutation and crossover. Final criteria that been consider is the objective function during operation of the technique. Some algorithm uses static objective function to achieve optimal solution.

However, there is also algorithms which uses reward or penalty to modify the objective function. This dynamic objective function allows the search process to be guided in the correct direction to obtain global optimal solution. All four criteria are used to determine the main trait for each algorithm then classified into the group as shown in Figure 1.

\section{POPULATION-BASED METAHEURISTIC SEARCH TECHNIQUES}

The basic operation and theory to obtain OPF have been discussed. This section will focus on the exploration and exploitation. Techniques on current popular algorithms such as Particle Swarm Optimization (PSO), Firefly Algorithm (FA), Artificial Bee Colony Algorithm (ABC), Ant Colony Optimization (ACO) and Differential Evolution Algorithm (DE). Those five algorithms are compared due to the versatility in optimization process in various problem and the development in various study and hybridization techniques.

\subsection{Particle Swarm Optimization Algorithm (PSO)}

PSO was developed by Kennedy and Eberhart in 1995 which is based on the behavior of bird and fish schooling in nature [9], [10]. PSO is widely used due to the details explanation in algorithm operation in their book 'Swarm Intelligent' [11]. This allows PSO to be easily hybridized with other algorithms to compensate with the PSO limitation when dealing with several problems. The movement of particles depend on stochastic component and deterministic component. This two main components did used to determine the current global best location and current best location. Since the movement is random, active comparison occurred and allowing global best solution can be obtained over time. The vector can be represented as:

$$
\begin{aligned}
& v_{i}^{t+1}=v_{i}^{t}+\alpha \epsilon_{1}\left[g^{*}-x_{i}^{t}\right]+\beta \epsilon_{2}\left[x_{i}^{*}-x_{i}^{t}\right] \\
& v_{i}^{t+1}=x_{i}^{t}+v_{i}^{t+1}
\end{aligned}
$$

$$
\text { where : } \begin{array}{ll}
x_{i} & \text { : Position of a particle } \\
\mathrm{Vi} & : \text { Velocity of a particle } \\
\epsilon_{1}, \epsilon_{2} & \begin{array}{l}
\text { : Random vectors that carry values } \\
\text { between } 0 \text { and } 1
\end{array} \\
\alpha, \beta & \text { : Learning parameters or } \\
& \text { acceleration constants, typically } \\
& \alpha \approx \beta \approx 2
\end{array}
$$

Based on (13) and (14), PSO consists of mutation and selection as whole. Crossover is not present in PSO algorithm. This allows a high exploration rate in order for fast convergence rate. Since no crossover happens during the process, it is high possible that the current global best solution has prematurely converged. 
However, there is possibility to achieve global optimal solution. The randomization for initial location allowing for the process take different route. Multiple random location will possibility lead to global optimal solution.

\subsection{Firefly Algorithm (FA)}

FA was developed by Xing-She Yang in 2008 which based on the flashing patterns and behavior of tropical fireflies [12]-[14]. The brightness of the flashing will be used for better exploration. The vector can be represented as:

$$
x_{i}^{t+1}=x_{i}^{t}+\beta_{0} e^{-\gamma r_{i / j}^{2}}\left(x_{j}^{t}-x_{i}^{t}\right)+\alpha \epsilon_{i}^{t}
$$

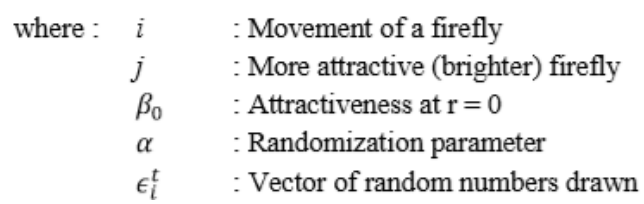

Based on (15), FA is easier to be implemented and flexible. The mutation that used from random numbers drawn from the Gaussian distribution and Levy flight allowing local and global search. The rate of searching can be controlled with randomization parameter. When compared with PSO, FA did not have global best solution which means FA did not undergo selection process.

However, FA uses ranking system which will be updated during the loops. The attraction feature is unique for FA which allows it to automatically classifies the solution into several groups [15]. This due to fact that the local attraction is stronger than the attraction from another location. The classification allows FA suitable to be used as multi objective problems and can be applied in various applications which are highly non-linear problems.

\subsection{Artificial Bee Colony Algorithm (ABC)}

$\mathrm{ABC}$ algorithm was developed by Karaboga in 2005 which is based on social behavior of bees [16]. $\mathrm{ABC}$ algorithm is divide into three group which are forager bees (employed), observer bees (onlooker) and scout bees. ABC did used the concentration of solution as their indicator which known as the amount of nectar intake from different food sources. Scouts and employed is used for mutation process to generate randomization then the solution will be selected by the onlooker. Employed bees will forage every food sources available with ratio of one bee per food source. When the food sources area been discard, forager bee will become scout to search for new food sources area by referring to the information given by onlooker bees. When compared to honey bee algorithm, $\mathrm{ABC}$ has advantage when the forager bees converted to scout directly. The vector for forager bees phase, observer bees phase and scout bee phase can be represented respectively as:

$$
\begin{aligned}
& v_{i j}=x_{i j}+\emptyset_{i j}\left(x_{i j}-x_{k j}\right) \\
& p_{i}=\frac{f i t_{i}}{\sum_{j=1}^{S_{N}} f i t_{j}} \\
& x_{i j}=x_{j}^{\min }+\left(x_{j}^{\max }-x_{j}^{\min }\right) \operatorname{rand}(0,1)
\end{aligned}
$$

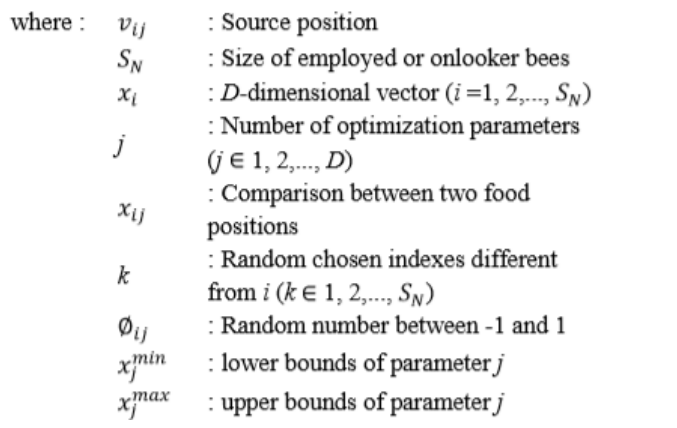


Based on (16)-(18), there is no crossover to maximize their randomization. This allowing the algorithm to adapt with tough optimization since the operation is simplify with the specific task which already been assigned for the particular roles [17], [18]. ABC in general have the ability to globally search which cause the convergence rate relatively slow and the exploitation ability is limited due to the absence of crossover process.

\subsection{Ant Colony Optimization Algortihm (ACO)}

ACO algorithm was developed by Dorigo in 1999 which based on social behavior of ant [19-21]. When compared to FA which using attraction, ACO used similar techniques as ABC which uses the concentration of route (quality of solutions). This concentration shows the quality of the solution which is generated for discrete combinatorial problems. The vector can be represented as:

$$
p_{i j}=\frac{\emptyset_{i j}^{\alpha} d_{i j}^{\beta}}{\sum_{i, j=1}^{n} \emptyset_{i j}^{\alpha} d_{i j}^{\beta}}
$$

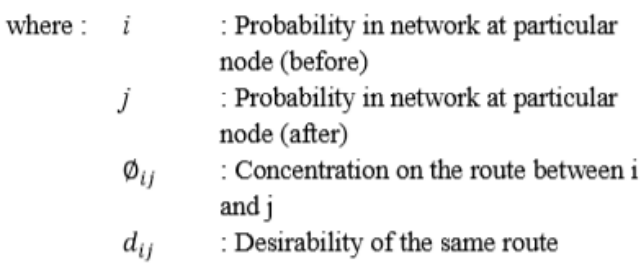

Based on (19), ACO did used evaporation over time features to avoid premature convergence. The main technique by ACO is the probability of choosing a route and the evaporation rate [22]. The best solution will display with high concentration of route due to the number of route used is higher due to traveling time is short, thus less amount of evaporated within that short period. This short term memory allowing the best route remain higher in concentration without causing any redundant from another local maximum which been discovered previously.

\subsection{Differential Evolution Algorithm (DE)}

DE algorithm developed by Rainer and Kenneth in 1997 which based on creating new off-spring from parent chromosomes instead of classical crossover or mutation [23]-[25]. DE did used crossover, mutation and selection. The unique of DE is the self-adaptive mechanism and selection process cause by their mutation scheme. DE used greedy selection process that provide slightly advantages of converging performance for the new and parent solution that win the selection [24], [26], [27]. DE can be stated into four stages which are initialization, mutation, crossover and selection.

During the initialization, the component can be represented as:

$$
x_{i, j}(0)=x_{j}^{L}+\operatorname{rand}(0,1)\left(x_{j}^{u}-x_{j}^{L}\right)
$$

where : $\operatorname{rand}(0,1)$ : Uniformly distributed random

$$
\text { number }
$$

$x_{j}^{u} \quad$ : parameter of upper bound

$x_{j}^{L} \quad$ : parameter of lower bound

$i \quad$ : Population members

$j \quad$ : Component of population

While the mutation phase, donor vector is created from the changes of population member which can be represent as:

$$
v_{i, j}(t+1)=x_{r_{1, j}}(t)+F\left(x_{r_{2, j}}(t)-x_{r_{3, j}}(t)\right)
$$


[14] X.-S. Yang, "Metaheuristic Optimization,” Cambridge Univ. UK, vol. 6, no. 2011, p. 15, 2011.

[15] T. Govindaraj and V. Tamildurai, "Firefly Algorithm for Optimal Power Flow Considering Control Variables," vol. 2, no. 2, pp. 1116-1121, 2014

[16] D. Karaboga, "An idea based on Honey Bee Swarm for Numerical Optimization," Tech. Rep. TR06, Erciyes Univ., no. TR06, p. 10, 2005.

[17] M. Ding, H. Chen, N. Lin, S. Jing, F. Liu, and X. Liang, "Dynamic population artificial bee colony algorithm for multiobjective optimal power flow," Saudi J. Biol. Sci., vol. 24, no. 3, pp. 703-710, 2017.

[18] L. Le Dinh, D. V. Ngoc, and P. Vasant, "Artificial Bee Colony Algorithm for Solving Optimal Power Flow Problem," vol. 2013, 2013.

[19] M. Dorigo, G. Di Caro, and L. M. Gambardella, “Ant algorithms for distributed discrete optimization," Artifical Life, vol. 5, pp. 137-172, 1999.

[20] J. Soares et al., “Ant Colony Search Algorithm for the Optimal Power Flow Problem,” pp. 1-8, 2011.

[21] K. Lenin, B. R. Reddy, and M. S. Kalavathi, "Optimal Power Flow using Ant Colony Search Algorithm to Evaluate Load Curtailment Incorporating Voltage Stability Margin Criterion,” vol. 3, no. 5, 2013.

[22] F. M. Tuaimah, "Ant Colony Optimization based Optimal Power Flow Analysis for the Iraqi Super High Voltage Grid," vol. 67, no. 11, pp. 13-18, 2013.

[23] R. Storn and K. Price, "Differential Evolution - A simple and efficient adaptive scheme for global optimization over continuous spaces," J. Glob. Optim., vol. 11, no. 4, pp. 341-359, 1997.

[24] A. A. A. El and E. M. A. Abido, "Optimal power flow using differential evolution algorithm," pp. 69-78, 2009.

[25] P. P. Biswas, P. N. Suganthan, R. Mallipeddi, and G. A. J. Amaratunga, "Engineering Applications of Artificial Intelligence Optimal power flow solutions using differential evolution algorithm integrated with effective constraint handling techniques," Eng. Appl. Artif. Intell., vol. 68, no. October 2017, pp. 81-100, 2018.

[26] A. Shrivastava and H. M. Siddiqui, "A Simulation Analysis of Optimal Power Flow using Differential Evolution Algorithm for IEEE-30 Bus System,” vol. 2, no. 3, pp. 50-57, 2014.

[27] R. K. Verma, H. Singh, and L. Srivastava, "Optimal Power Flow Using Differential Evolution Algorithm With Conventional Weighted Sum Method,” vol. 2, no. 3, pp. 681-685, 2012. 